\title{
Effect of Priming Treatments to Enhance Seed Quality of Naturally Aged Seed of Forage Sorghum
}

\author{
Nisha $^{1 *}$, S. S. Jakhar ${ }^{1}$, Axay Bhuker ${ }^{1}$ and Satpal ${ }^{2}$ \\ ${ }^{1}$ Department of Seed Science \& Technology, ${ }^{2}$ Forage Section, Department of Genetics \& \\ Plant Breeding, CCS Haryana Agricultural University, Hisar-125 004 (Haryana), India \\ *Corresponding author
}

\section{A B S T R A C T}

K e y w o r d s
Forage sorghum,
emergence time,
emergence index,
seedling
establishment and
seed priming
Article Info
Accepted:
15 April 2020
Available Online:
10 May 2020

\section{Keywords}

Forage sorghum, emergence time, emergence index establishment and priming

Article Info

Accepted:

Available Online:

10 May 2020

\begin{abstract}
The present study was undertaken to find out the effect of priming treatments to enhance seed quality of naturally aged seeds of forage sorghum varieties. The experiment was conducted at Research Farm and Seed technology laboratory, Department of Seed Science \& Technology, CCS Haryana Agricultural University, Hisar (Haryana), India during kharif season, 2018. Three naturally aged seed lots (fresh, one year old and two year old) of two single-cut forage sorghum varieties (HC 136 and $\mathrm{HJ} 541$ ) were tested with five priming treatments $\left[\mathrm{T}_{1}\right.$ : Untreated, $\mathrm{T}_{2}$ : Hydration-dehydration (6 hrs.), $\mathrm{T}_{3}$ : Hydration-dehydration $(6 \mathrm{hrs}$. $)+0.25 \%$ thiram treatment, $\mathrm{T}_{4}$ : Hydration with $\mathrm{GA}_{3} @ 50$ ppm for 6 hrs, $\mathrm{T}_{5}$ : Hydration with sodium molybdate @ $500 \mathrm{ppm}$ for $6 \mathrm{hrs}$.]. Among varieties, HJ 541 performed better than HC 136 with each priming treatments. Among naturally aged seed lots, maximum improvement was observed in freshly harvested seed lot $\left(\mathrm{L}_{1}\right)$ while, minimum improvement was found in two year old seed lot $\left(\mathrm{L}_{3}\right)$ when treated with different treatments. Among priming treatments, $\mathrm{T}_{4}$ i.e. hydration with $\mathrm{GA}_{3} @ 50 \mathrm{ppm}$ for $6 \mathrm{hrs}$ showed highest improvement in speed of emergence in both the varieties and each seed lot followed by Hydration - dehydration + $0.25 \%$ Thiram $\left(\mathrm{T}_{3}\right)$ and Hydration with sodium molybdate $\left(\mathrm{T}_{5}\right)$. Based on the results, it was concluded that fresh seed lot of HJ 541 variety with priming treatment of hydration with $\mathrm{GA}_{3} @ 50$ ppm for 6 hrs proved better on majority of the seed viability and vigour parameters.
\end{abstract}

\section{Introduction}

Sorghum [Sorghum bicolor (L.) Moench], belonging to family Poaceae, is an important kharif season crop which is widely grown to meet the green fodder, dry fodder and feed requirement of the livestock (Satpal et al., 2015). Besides this, sorghum can be used as an alternate food, source of raw materials bioethanol industry. The nutritional content of sorghum is comparable to maize and rice, having protein content to the tune of $10-11 \%$.

Sorghum has a high nutrient content that is 339 calories and $11.3 \%$ protein/100 grams of seeds (USDA, 2011). India faces a net deficit of $36 \%$ and $11 \%$ of green fodder and dry fodder, respectively (Tokas et al., 2019). 
To reduce the demand and supply gap, the production and productivity of fodder crops needs to be enhanced. Sorghum being a very importance fodder crop can help in bridging this gap by providing high productivity of green fodder in summer and kharif season. But the availability of quality of forage sorghum varieties is again a cause of concern to the dairy farmers. As per estimates, only 25-30 per cent of required quantity of quality seed is available in cultivated fodders in India.

Presently, the seed demand of cultivated forages is increasing tremendously. Now, with the development of a number of improved and high yielding varieties in forage crops, it has become important that quality seed should be readily available and supplied to the farmers at reasonable price. As, it has been affirmed that utilization of high value seed improved the yield by 15-20 per cent (Sindhawani, 1991). Seed possesses maximum viability and vigour at physiological maturity (Meena et al., 1994), thereafter, seeds gradually aged and decline in viability and vigour. Seed deterioration leads to reduction in seed quality, performance and stand establishment. Higher moisture content along with high temperature of storage environment, the sooner is the loss of viability (Abbas and Lovato, 1999).

Ageing causes deterioration in all vital cellular components of seed thereby advance loss of viability. Lipid auto-oxidation has also been proposed to be one of the causes of seed ageing (McDonald, 1986) which involve the production of free radicals. According to Bortey et al., (2016), seed storage period may affect the viability of seeds, as the reduction in seed viability is directly proportional to the increase of time. This is because it allows the ripening embryo storage period and further accumulation of food that lasts for storage before germinating, these activities led to an increase in the metabolic processes in the seed. Such problems convey severe threat to agriculture; hence require management to sustain viability and vigour. Seed priming is a pre-sowing treatment which leads to a physiological state that enables seed to germinate more efficiently. The majority of seed treatments are based on seed imbibition allowing the seeds to go through the first reversible stage of germination but do not allow radical protrusion through the seed coat. Seeds keeping their desiccation tolerance are then dehydrated and can be stored until final sowing. During subsequent germination, primed seeds exhibit a faster and more synchronized germination and young seedlings are often more vigorous and resistant to abiotic stresses than seedlings obtained from unprimed seeds. Keeping in view, the importance of seed priming, the experiment was conducted to study the effect of priming treatments to enhance seed quality of naturally aged seed of forage sorghum

\section{Materials and Methods}

The three factor experiment was conducted during rainy (kharif) season of 2018 at Research Farm, Department of Seed Science \& Technology, CCS Haryana Agricultural University, Hisar (Haryana), India (29 $10^{\prime} \mathrm{N}$ of $75^{\circ} 46^{\prime} \mathrm{E}$, at an average elevation of 215.2 $\mathrm{m}$ above mean sea level). The site has semiarid and sub-tropical climate with hot dry summer and severe cold winter.

Average annual rainfall is about $450 \mathrm{~mm}, 75$ per cent of which is received in three months, from July to September during south-west monsoon. Fig. 1 represents the weekly weather parameters i.e. temperature ${ }^{\circ} \mathrm{C}$ (a), relative humidity (\%) (b), bright sunshine (h) (c) and rainfall (mm) (d) during the study. The soil of the experimental field was sandy loam in texture, slightly alkaline in reaction (pH 7.3). 
The varieties used in the experiment were $\mathrm{HC}$ 136 and HJ 541. The three factor experiment (factor 1: two varieties, factor 2: Three seed lots and factor 3: Five priming treatment) was carried out in three replicates with 100 seeds of each seed lots of each variety include fresh $\left(\mathrm{L}_{1}\right)$, one year old $\left(\mathrm{L}_{2}\right)$ and two year old $\left(\mathrm{L}_{3}\right)$ seed stored under ambient conditions were sown in a factorial randomized block design. The date of sowing of the experiment was 10 July 2018. For this experiment, natural aged seeds of both the varieties were treated with following priming treatments. $\mathrm{T}_{1}$ : Untreated (control), $\mathrm{T}_{2}$ : Hydration-dehydration (6 hrs.), $\mathrm{T}_{3}$ : Hydration- dehydration $(6 \mathrm{hrs}$.) $+0.25 \%$ thiram treatment, $\mathrm{T}_{4}$ : Hydration with $\mathrm{GA}_{3}(50$ ppm for $6 \mathrm{hrs}$.), $\mathrm{T}_{5}$ : Hydration with sodium molybdate $(500 \mathrm{ppm}$ for $6 \mathrm{hrs}$.). The following observations were recorded in the trial.

\section{Speed of emergence index}

On each day, the number of seedlings emerged were counted and continued up to the seedling establishment and field emergence index also termed as the speed of emergence was calculated by the method as described by Maguire (1962).

Speed of $\quad$ emergence index
$=\frac{\text { Noofseedlings emerged }}{\text { First day of sowing }}+\cdots+\frac{\text { Noof seedlings emerged }}{\text { Day of lastcount(15th) }}$

\section{Seedling establishment (SET \%)}

When the seedling emergence was accomplished or there was no further increase in total seedling emergence, the seedling establishment was evaluated by counting the total number of seedlings (up to 15 days).

\section{Mean emergence time (days)}

The mean emergence time was observed for each treatment combination using the formula cited by Ellis and Robert (1977)

Mean Emergence Time $=\sum n t / \sum N$

Where,

$\mathrm{n}=$ number of seeds newly

germinated at time ' $\mathrm{t}$ '

$\mathrm{t}=$ days from sowing

$N \quad=\quad$ Final emergence of seedlings

\section{Results and Discussion}

Data presented in Table no 2 reveal that, all the treatments improved speed of emergence considerably in all the seed lots and varieties. However, freshly harvested seed lot $\left(\mathrm{L}_{1}\right)$ showed highest improvement whereas two year old seed lot $\left(\mathrm{L}_{3}\right)$ showed lowest improvement. The variety $\mathrm{HJ} 541 \quad\left(\mathrm{~V}_{2}\right)$ performed better than $\mathrm{HC} 136\left(\mathrm{~V}_{1}\right)$ with each priming treatments. The treatment $\mathrm{GA}_{3}\left(\mathrm{~T}_{4}\right)$ showed highest improvement in speed of emergence in two varieties and each seed lot followed by Hydration - dehydration +0.25 $\%$ Thiram $\left(\mathrm{T}_{3}\right)$ and Hydration with sodium molybdate $\left(\mathrm{T}_{5}\right)$.

The results confirmed the earlier findings by Soltani et al., 2009. Data presented in Table no 2 further reveal that improvement in seedling establishment was noticed in both varieties and seed lots of sorghum. However, maximum improvement was observed in freshly harvested seed lot $\left(\mathrm{L}_{1}\right)$ while, minimum improvement was found in two year old seed lot $\left(\mathrm{L}_{3}\right)$ when treated with different treatments. Among varieties, highest improvement was recorded in variety HJ 541 $\left(\mathrm{V}_{2}\right)$ followed by $\mathrm{HC} 136\left(\mathrm{~V}_{1}\right)$, when treated with different priming treatments. Treatment $\left(\mathrm{T}_{4}\right)$ hydration with $\mathrm{GA}_{3}$ (50 ppm for $6 \mathrm{hrs}$ ) showed best results in all the seed lots and varieties followed by Hydration-dehydration $+0.25 \%$ Thiram treatment $\left(\mathrm{T}_{3}\right)$ and Hydration- dehydration treatment $\left(\mathrm{T}_{2}\right)$, respectively. 
Similar finding was reported in Brassica compestris by Verma et al., 2003, in coriander by Kumar, 2007, in wheat by Singh, 2009. Bobak et al., (2015) suggested that seed ageing for higher duration could significantly decreased seed quality of corn seed. Using seed enhancement treatments like seed priming or application of phytohormone could improve aged and non-aged seed performance especially for higher aged seed. Data presented in Table 3 reveal that mean emergence time (MET) was decreased in all the seed lots and variety with all the treatments. However, fresh year seed lot $\left(\mathrm{L}_{1}\right)$ show highest decrease in MET while, lowest decrease was observed in two year old seed lot $\left(\mathrm{L}_{3}\right)$ when treated with different priming treatments. Among varieties HJ $541\left(\mathrm{~V}_{2}\right)$ show highest decrease in MET when treated with different treatments. The application of $\mathrm{GA}_{3}$ treatment $\left(\mathrm{T}_{4}\right)$ showed highest decrease in MET followed by Hydration-dehydration + $0.25 \%$ Thiram treatment $\left(\mathrm{T}_{3}\right)$ and lowest decrease with Hydration-dehydration $\left(\mathrm{T}_{2}\right)$. Similar finding were also reported in caper seeds by Pascual et al., (2006).

Table.1 Effect of pre-sowing treatments on speed of emergence of natural aged seed of sorghum

Table.1 (a) Interaction between varieties and treatments

\begin{tabular}{|l|c|c|c|c|c|c|}
\hline \multirow{2}{*}{ Varieties } & \multicolumn{5}{|c|}{ Treatments } & Mean \\
\cline { 2 - 7 } & $\mathbf{T}_{\mathbf{1}}$ & $\mathbf{T}_{\mathbf{2}}$ & $\mathbf{T}_{\mathbf{3}}$ & $\mathbf{T}_{\mathbf{4}}$ & $\mathbf{T}_{\mathbf{5}}$ & \\
\hline HC 136 $\left(\mathbf{V}_{\mathbf{1}}\right)$ & 5.95 & 6.00 & 6.65 & 6.89 & 6.32 & $\mathbf{6 . 3 6}$ \\
\hline HJ 541 $\left(\mathbf{V}_{\mathbf{2}}\right)$ & 7.02 & 7.28 & 7.76 & 8.45 & 7.60 & $\mathbf{7 . 6 2}$ \\
\hline Mean & $\mathbf{6 . 4 8}$ & $\mathbf{6 . 6 4}$ & $\mathbf{7 . 2 1}$ & $\mathbf{7 . 6 7}$ & $\mathbf{6 . 9 6}$ & \\
\hline
\end{tabular}

$\mathrm{CD}(\mathrm{P}=0.05) \mathrm{V}=0.017, \mathrm{~T}=0.027, \mathrm{~V} \times \mathrm{T}=0.038$

Table.1 (b) Interaction between varieties and seed lots

\begin{tabular}{|l|c|c|c|c|}
\hline \multirow{2}{*}{ Varieties } & \multicolumn{3}{|c|}{ Seed lots } & Mean \\
\cline { 2 - 5 } & Fresh year $\left(\mathbf{L}_{\mathbf{1}}\right)$ & One year old $\left(\mathbf{L}_{\mathbf{2}}\right)$ & Two year old $\left(\mathbf{L}_{\mathbf{3}}\right)$ & \\
\hline HC 136 $\left(\mathbf{V}_{\mathbf{1}}\right)$ & 8.83 & 7.01 & 3.26 & 6.36 \\
\hline HJ 541 $\left(\mathbf{V}_{\mathbf{2}}\right)$ & 8.78 & 7.95 & 6.12 & 7.62 \\
\hline Mean & 8.80 & 7.48 & 4.69 & \\
\hline
\end{tabular}

$\mathrm{CD}(\mathrm{P}=0.05) \mathrm{V}=0.017, \mathrm{~L}=0.021, \mathrm{~V} \times \mathrm{L}=0.030$

Table.1 (c) Interaction between treatments and seed lots

\begin{tabular}{|l|c|c|c|c|c|c|}
\hline \multirow{2}{*}{ Seed lots } & \multicolumn{5}{|c|}{ Treatments } & \multirow{2}{*}{ Mean } \\
\cline { 1 - 7 } & $\mathbf{T}_{\mathbf{1}}$ & $\mathbf{T}_{\mathbf{2}}$ & $\mathbf{T}_{\mathbf{3}}$ & $\mathbf{T}_{\mathbf{4}}$ & $\mathbf{T}_{\mathbf{5}}$ & \\
\hline Fresh seed $\left(\mathbf{L}_{\mathbf{1}}\right)$ & 7.99 & 8.43 & 9.20 & 9.93 & 8.46 & $\mathbf{8 . 8 0}$ \\
\hline One year old $\left(\mathbf{L}_{\mathbf{2}}\right)$ & 6.88 & 6.90 & 7.55 & 8.25 & 7.84 & $\mathbf{7 . 4 8}$ \\
\hline Two year old $\left(\mathbf{L}_{\mathbf{3}}\right)$ & 4.58 & 4.59 & 4.89 & 4.83 & 4.58 & $\mathbf{4 . 6 9}$ \\
\hline Mean & $\mathbf{6 . 4 8}$ & $\mathbf{6 . 6 4}$ & $\mathbf{7 . 2 1}$ & $\mathbf{7 . 6 7}$ & $\mathbf{6 . 9 6}$ & \\
\hline
\end{tabular}

$\mathrm{CD}(\mathrm{P}=0.05) \mathrm{L}=0.021, \mathrm{~T}=0.027, \mathrm{~L} \times \mathrm{T}=0.047, \mathrm{~V} \times \mathrm{L} \times \mathrm{T}=0.066$ 
Table.1 (d) Effect of pre-sowing treatments on speed of emergence of different seed lots and varieties of sorghum

\begin{tabular}{|l|c|c|c|c|c|}
\hline \multirow{2}{*}{ Treatments } & \multicolumn{3}{|c|}{ Effect on seed lots } & \multicolumn{2}{|c|}{ Effect on varieties } \\
\cline { 2 - 6 } & $\begin{array}{c}\text { Fresh seed } \\
\left(\mathbf{L}_{\mathbf{1}}\right)\end{array}$ & $\begin{array}{c}\text { One year old } \\
\left(\mathbf{L}_{\mathbf{2}}\right)\end{array}$ & $\begin{array}{c}\text { Two year old } \\
\left(\mathbf{L}_{\mathbf{3}}\right)\end{array}$ & $\begin{array}{c}\text { HC 136 } \\
\left(\mathbf{V}_{\mathbf{1}}\right)\end{array}$ & $\begin{array}{c}\text { HJ 541 } \\
\left(\mathbf{V}_{\mathbf{2}}\right)\end{array}$ \\
\hline $\mathbf{T}_{\mathbf{2}}-\mathbf{T}_{\mathbf{1}}$ & 0.44 & 0.01 & 0.01 & 0.05 & 0.26 \\
\hline $\mathbf{T}_{\mathbf{3}}-\mathbf{T}_{\mathbf{1}}$ & 1.21 & 0.67 & 0.31 & 0.70 & 0.74 \\
\hline $\mathbf{T}_{\mathbf{4}}-\mathbf{T}_{\mathbf{1}}$ & 1.94 & 1.37 & 0.25 & 0.94 & 1.43 \\
\hline $\mathbf{T}_{\mathbf{5}}-\mathbf{T}_{\mathbf{1}}$ & 0.46 & 0.95 & 0.01 & 0.37 & 0.58 \\
\hline
\end{tabular}

$\mathrm{V}_{1}=\mathrm{HC} 136, \mathrm{~V}_{2}=\mathrm{HJ} 541, \mathrm{~T}_{1}$-Untreated (control), $\mathrm{T}_{2}$-Hydration-dehydration (6 hrs), $\mathrm{T}_{3}$-Hydration-dehydration $(6 \mathrm{hrs})+0.25 \%$ thiram, $\mathrm{T}_{4}$-Hydration with $\mathrm{GA}_{3}(50 \mathrm{ppm}$ for $6 \mathrm{hrs}), \mathrm{T}_{5}$-Hydration with sodium molybdate (500 ppm for $6 \mathrm{hrs}$ ).

Table.2 Effect of pre-sowing treatments on seedling establishment percentage of natural aged seed of sorghum

Table.2 (a) Interaction between varieties and treatments

\begin{tabular}{|l|c|c|c|c|c|c|}
\hline \multirow{2}{*}{ Varieties } & \multicolumn{5}{|c|}{ Treatments } & Mean \\
\cline { 1 - 7 } & $\mathbf{T}_{\mathbf{1}}$ & $\mathbf{T}_{\mathbf{2}}$ & $\mathbf{T}_{\mathbf{3}}$ & $\mathbf{T}_{\mathbf{4}}$ & $\mathbf{T}_{\mathbf{5}}$ & \\
\hline HC 136 $\left(\mathbf{V}_{\mathbf{1}}\right)$ & 46.00 & 48.00 & 49.78 & 55.11 & 46.11 & $\mathbf{4 9 . 0 0}$ \\
& $(41.81)$ & $(43.16)$ & $(45.77)$ & $(46.67)$ & $(42.097)$ & $\mathbf{( 4 3 . 7 6 2 )}$ \\
\hline HJ 541 $\left(\mathbf{V}_{\mathbf{2}}\right)$ & 57.56 & 59.00 & 62.00 & 66.44 & 60.22 & $\mathbf{6 1 . 0 4}$ \\
& $(49.435)$ & $(50.294)$ & $(52.768)$ & $(53.566)$ & $(51.253)$ & $\mathbf{( 5 1 . 4 6 3 )}$ \\
\hline Mean & $\mathbf{5 1 . 7 8}$ & $\mathbf{5 3 . 5 0}$ & $\mathbf{5 5 . 8 9}$ & $\mathbf{6 0 . 7 8}$ & $\mathbf{5 3 . 1 7}$ & \\
& $\mathbf{( 4 5 . 6 2 2})$ & $\mathbf{( 4 6 . 7 2 5})$ & $\mathbf{( 4 8 . 9 2 3 )}$ & $\mathbf{( 5 0 . 1 2 0})$ & $\mathbf{( 4 6 . 6 7 5 )}$ & \\
\hline
\end{tabular}

$\mathrm{CD}(\mathrm{P}=0.05) \mathrm{V}=0.124, \mathrm{~T}=0.197, \mathrm{~V} \times \mathrm{T}=0.278$

Table.2 (b) Interaction between varieties and seed lots

\begin{tabular}{|l|c|c|c|c|}
\hline \multirow{2}{*}{ Varieties } & \multicolumn{3}{|c|}{ Seed lots } & Mean \\
\cline { 2 - 5 } & Fresh year $\left(\mathbf{L}_{\mathbf{1}}\right)$ & One year old $\left(\mathbf{L}_{\mathbf{2}}\right)$ & Two year old $\left(\mathbf{L}_{\mathbf{3}}\right)$ & \\
\hline HC 136 $\left(\mathbf{V}_{\mathbf{1}}\right)$ & $70.80(57.37)$ & $60.60(50.80)$ & $15.60(23.13)$ & $\mathbf{4 9 . 0 0}(\mathbf{4 3 . 7 6})$ \\
\hline HJ 541 $\left(\mathbf{V}_{\mathbf{2}}\right)$ & $72.73(59.57)$ & $65.47(54.01)$ & $44.93(40.82)$ & $\mathbf{6 1 . 0 4}(\mathbf{5 1 . 4 6})$ \\
\hline Mean & $\mathbf{7 1 . 7 7}(\mathbf{5 8 . 4 7})$ & $\mathbf{6 3 . 0 3}(\mathbf{5 2 . 4 0})$ & $\mathbf{3 0 . 2 7}(\mathbf{3 1 . 9 7})$ & \\
\hline $\mathrm{CD}(\mathrm{P}=0.05) \mathrm{V}=0.124, \mathrm{~L}=0.152, \mathrm{~V} \times \mathrm{L}=0.216$ & & \\
\hline
\end{tabular}


Table.2 (c) Interaction between treatments and seed lots

\begin{tabular}{|l|c|c|c|c|c|c|}
\hline \multirow{2}{*}{ Seed lots } & \multicolumn{5}{|c|}{ Treatments } & Mean \\
\cline { 2 - 7 } & $\mathbf{T}_{\mathbf{1}}$ & $\mathbf{T}_{\mathbf{2}}$ & $\mathbf{T}_{\mathbf{3}}$ & $\mathbf{T}_{\mathbf{4}}$ & $\mathbf{T}_{\mathbf{5}}$ & \\
\hline Fresh seed $\left(\mathbf{L}_{\mathbf{1}}\right)$ & 68.93 & 70.50 & 72.00 & 77.50 & 69.00 & $\mathbf{7 1 . 7 7}$ \\
& $(56.66)$ & $(57.08)$ & $(60.03)$ & $(61.66)$ & $(56.88)$ & $\mathbf{( 5 8 . 4 6 )}$ \\
\hline One year old $\left(\mathbf{L}_{\mathbf{2}}\right)$ & 59.00 & 61.50 & 64.67 & 67.50 & 62.50 & $\mathbf{6 3 . 0 3}$ \\
& $(50.17)$ & $(51.63)$ & $(53.51)$ & $(54.47)$ & $(52.24)$ & $\mathbf{( 5 2 . 4 0})$ \\
\hline Two year old $\left(\mathbf{L}_{\mathbf{3}}\right)$ & 26.50 & 28.50 & 31.00 & 37.33 & 28.00 & $\mathbf{3 0 . 2 7}$ \\
& $(30.03)$ & $(31.46)$ & $(33.23)$ & $(34.23)$ & $(30.90)$ & $\mathbf{( 3 1 . 9 7 )}$ \\
\hline Mean & $\mathbf{5 1 . 7 8}$ & $\mathbf{5 3 . 5 0}$ & $\mathbf{5 5 . 8 9}$ & $\mathbf{6 0 . 7 8}$ & $\mathbf{5 3 . 1 7}$ & \\
& $\mathbf{4 5 . 6 2}$ & $\mathbf{( 4 6 . 7 3 )}$ & $\mathbf{( 4 8 . 9 2 )}$ & $\mathbf{( 5 0 . 1 2 )}$ & $\mathbf{( 4 6 . 6 8 )}$ & \\
\hline
\end{tabular}

$\mathrm{CD}(\mathrm{P}=0.05) \mathrm{L}=0.152, \mathrm{~T}=0.197, \mathrm{~L} \times \mathrm{T}=0.341, \mathrm{~V} \times \mathrm{L} \times \mathrm{T}=0.482$

Table.2 (d) Effect of pre-sowing treatments on seedling establishment percentage of different seed lots and varieties of sorghum

\begin{tabular}{|l|c|c|c|c|c|}
\hline \multirow{2}{*}{ Treatments } & \multicolumn{3}{|c|}{ Effect on seed lots } & \multicolumn{2}{c|}{ Effect on varieties } \\
\cline { 2 - 6 } & $\begin{array}{c}\text { Fresh seed } \\
\text { (L1) }\end{array}$ & $\begin{array}{c}\text { One year old } \\
\text { (L2) }\end{array}$ & $\begin{array}{c}\text { Two year old } \\
(\mathbf{L 3})\end{array}$ & $\begin{array}{c}\text { HC 136 } \\
\text { (V1) }\end{array}$ & $\begin{array}{c}\text { HJ 541 } \\
(\mathbf{V} 2)\end{array}$ \\
\hline $\mathbf{T}_{\mathbf{2}}-\mathbf{T}_{\mathbf{1}}$ & 0.418 & 1.459 & 1.433 & 1.347 & 0.859 \\
\hline $\mathbf{T}_{\mathbf{3}}-\mathbf{T}_{\mathbf{1}}$ & 3.372 & 3.334 & 3.198 & 3.269 & 3.333 \\
\hline $\mathbf{T}_{\mathbf{4}}-\mathbf{T}_{\mathbf{1}}$ & 5.000 & 4.294 & 4.203 & 4.866 & 4.131 \\
\hline $\mathbf{T}_{\mathbf{5}}-\mathbf{T}_{\mathbf{1}}$ & 0.221 & 2.07 & 0.869 & 0.289 & 1.818 \\
\hline
\end{tabular}

$\mathrm{V}_{1}=\mathrm{HC} 136, \mathrm{~V}_{2}=\mathrm{HJ} 541, \mathrm{~T}_{1}$-Untreated (control), $\mathrm{T}_{2}$-Hydration-dehydration (6 hrs), $\mathrm{T}_{3}$-Hydration-dehydration $(6 \mathrm{hrs})+0.25 \%$ thiram, $\mathrm{T}_{4}$-Hydration with $\mathrm{GA}_{3}(50 \mathrm{ppm}$ for $6 \mathrm{hrs}), \mathrm{T}_{5}$-Hydration with sodium molybdate (500 ppm for 6 hrs).

Table.3 Effect of pre-sowing treatments on mean emergence time of natural aged seed of sorghum

Table.3 (a) Interaction between varieties and treatments

\begin{tabular}{|l|c|c|c|c|c|c|}
\hline \multirow{2}{*}{ Varieties } & \multicolumn{5}{|c|}{ Treatments } & \multirow{2}{*}{ Mean } \\
\cline { 2 - 7 } & $\mathbf{T}_{\mathbf{1}}$ & $\mathbf{T}_{\mathbf{2}}$ & $\mathbf{T}_{\mathbf{3}}$ & $\mathbf{T}_{\mathbf{4}}$ & $\mathbf{T}_{\mathbf{5}}$ & \\
\hline HC 136 $\left(\mathbf{V}_{\mathbf{1}}\right)$ & 6.750 & 6.670 & 6.687 & 6.634 & 6.730 & $\mathbf{6 . 7 0 6}$ \\
\hline HJ 541 $\left(\mathbf{V}_{\mathbf{2}}\right)$ & 6.363 & 6.323 & 6.253 & 6.161 & 6.323 & $\mathbf{6 . 1 9 5}$ \\
\hline Mean & $\mathbf{6 . 5 5 7}$ & $\mathbf{6 . 5 2 7}$ & $\mathbf{6 . 4 7 0}$ & $\mathbf{6 . 3 9 8}$ & $\mathbf{6 . 5 2 7}$ & \\
\hline
\end{tabular}

$\mathrm{CD}(\mathrm{P}=0.05) \mathrm{V}=0.008, \mathrm{~T}=0.013, \mathrm{~V} \times \mathrm{T}=0.018$ 
Table.3 (b) Interaction between varieties and seed lots

\begin{tabular}{|l|c|c|c|c|}
\hline \multirow{2}{*}{ Varieties } & \multicolumn{3}{|c|}{ Seed lots } & \multirow{2}{*}{ Mean } \\
\cline { 2 - 4 } & Fresh year $\left(\mathbf{L}_{\mathbf{1}}\right)$ & One year old $\left(\mathbf{L}_{\mathbf{2}}\right)$ & Two year old $\left(\mathbf{L}_{3}\right)$ & \\
\hline HC 136 $\left(\mathbf{V}_{\mathbf{1}}\right)$ & 5.761 & 6.659 & 7.699 & $\mathbf{6 . 7 0 6}$ \\
\hline HJ 541 $\left(\mathbf{V}_{\mathbf{2}}\right)$ & 5.295 & 6.233 & 7.327 & $\mathbf{6 . 1 9 5}$ \\
\hline Mean & $\mathbf{5 . 5 2 8}$ & $\mathbf{6 . 4 4 6}$ & $\mathbf{7 . 5 1 3}$ & \\
\hline $\mathrm{CD}(\mathrm{P}=0.05) \mathrm{V}=0.008, \mathrm{~L}=0.010, \mathrm{~V}$ x L=0.014 & & &
\end{tabular}

Table.3 (c) Interaction between treatments and seed lots

\begin{tabular}{|l|c|c|c|c|c|c|}
\hline \multirow{2}{*}{ Seed lots } & \multicolumn{5}{|c|}{ Treatments } & \multirow{2}{*}{ Mean } \\
\cline { 1 - 6 } & $\mathbf{T}_{\mathbf{1}}$ & $\mathbf{T}_{\mathbf{2}}$ & $\mathbf{T}_{\mathbf{3}}$ & $\mathbf{T}_{\mathbf{4}}$ & $\mathbf{T}_{\mathbf{5}}$ & \\
\hline Fresh seed $\left(\mathbf{L}_{\mathbf{1}}\right)$ & 5.613 & 5.573 & 5.490 & 5.388 & 5.573 & $\mathbf{5 . 5 2 8}$ \\
\hline One year old $\left(\mathbf{L}_{\mathbf{2}}\right)$ & 6.508 & 6.475 & 6.420 & 6.355 & 6.473 & $\mathbf{6 . 4 4 6}$ \\
\hline Two year old $\left(\mathbf{L}_{\mathbf{3}}\right)$ & 7.548 & 7.532 & 7.500 & 7.450 & 7.533 & $\mathbf{7 . 3 5 4}$ \\
\hline Mean & $\mathbf{6 . 5 5 7}$ & $\mathbf{6 . 5 2 7}$ & $\mathbf{6 . 4 7 0}$ & $\mathbf{6 . 3 9 8}$ & $\mathbf{6 . 5 2 7}$ & \\
\hline
\end{tabular}

$\mathrm{CD}(\mathrm{P}=0.05) \mathrm{L}=0.010, \mathrm{~T}=0.013, \mathrm{~L} \times \mathrm{T}=0.022, \mathrm{~V} \times \mathrm{Lx} \mathrm{T}=0.055$

Table.3 (d) Effect of pre-sowing treatments on mean emergence time of different seed lots and varieties of sorghum

\begin{tabular}{|l|c|c|c|c|c|}
\hline \multirow{2}{*}{ Treatments } & \multicolumn{3}{|c|}{ Effect on seed lots } & \multicolumn{2}{c|}{ Effect on varieties } \\
\cline { 2 - 6 } & Fresh seed & One year old & Two year old & HC 136 & HJ 541 \\
& $\left(\mathbf{L}_{\mathbf{1}}\right)$ & -0.033 & -0.016 & -0.02 & -0.04 \\
\hline $\mathbf{T}_{\mathbf{2}}-\mathbf{T}_{\mathbf{1}}$ & -0.04 & -0.088 & -0.048 & -0.063 & -0.11 \\
\hline $\mathbf{T}_{\mathbf{3}}-\mathbf{T}_{\mathbf{1}}$ & -0.123 & -0.153 & -0.098 & -0.116 & -0.202 \\
\hline $\mathbf{T}_{\mathbf{4}}-\mathbf{T}_{\mathbf{1}}$ & -0.225 & $\left.-\mathbf{L}_{\mathbf{2}}\right)$ & -0.02 & -0.04 \\
\hline $\mathbf{T}_{\mathbf{5}}-\mathbf{T}_{\mathbf{1}}$ & -0.04 & -0.035 & -0.035 & -0.02 & \\
\hline
\end{tabular}

$\mathrm{V}_{1}=\mathrm{HC}$ 136, $\mathrm{V}_{2}=\mathrm{HJ} 541, \mathrm{~T}_{1}$-Untreated (control), $\mathrm{T}_{2}$-Hydration-dehydration (6 hrs), $\mathrm{T}_{3}$-Hydration-dehydration $(6 \mathrm{hrs})+0.25 \%$ thiram, $\mathrm{T}_{4}$-Hydration with $\mathrm{GA}_{3}(50 \mathrm{ppm}$ for $6 \mathrm{hrs}), \mathrm{T}_{5}$-Hydration with sodium molybdate (500 ppm for $6 \mathrm{hrs}$ )

Priming of the seeds with different treatments was found effective to enhance the seed value in fresh as well as marginal seed lot i.e. one year seed lot. Application of $\mathrm{GA}_{3}(50 \mathrm{ppm}$ for $6 \mathrm{hrs}$.) was found to be the best priming treatment for improving the quality of seed followed by hydration- dehydration $(6 \mathrm{hrs}$. $)+$ $0.25 \%$ thiram treatments. All the priming treatments indicated maximum effect on $\mathrm{HJ}$ 541 followed by HC 136. 


\section{Acknowledgement}

Ms. Nisha, the first author is thankful to Department of Seed Science \& Technology, CCS HAU Hisar for providing necessary facilities to carry out the experiment during her M.Sc. (Agriculture) in Seed Science \& Technology. Thanks are also due to Forage Section, Department of Genetics \& Plant Breeding, CCS HAU, Hisar for providing seeds of sorghum varieties to carry out this research work.

\section{References}

Abbas, E.J. and Lovato, A. 1999. Effect of seed storage temperature and relative humidity on maize (Zea mays L.) seed viability and vigour. Seed Sci. and Technol. 27: 101-114.

Bobak, S.A. Parviz, N.K. and Ansari, W.M. 2015. An assessment of the effects of seed ageing, application of phytohormone and $\mathrm{KNO}_{3}$ on aged corn seeds. African Journal of Agronomy. 3(2): 235-243.

Bortey, H. M., Sadia, A. O. and Asibuo, J. Y. 2016. Influence of seed storage techniques on germinability and storability of cowpea (Vigna unguiculata (L.) Walp). Journal Agricultural Science. 8(10): 241-248. https://doi.org/10.5539/jas.v8n10p241.

Ellis, R.H. and Roberts, E.H. 1977. A revised seed viability monographs for onion Seed Res. 5:93-103.

Kumar, A. 2007. Seed quality assessment in naturally aged seed lot of coriander (Coriandrum sativum L.) M. Sc. Thesis CCS HAU, Hisar, Haryana, India.

Maguire, J.D. 1962. Speed of germination Aid in selection and evolution for seedling emergence and vigour. Crop Sci. 2: 176-177

McDonald, M.B. and Wilson, D.O. 1986. The lipid peroxidation model of seed deterioration. Seed Sci and Technol, 14:259-268.

Meena, R.A. Rathinavel, K. and Singh, P. 1994. Seed development and maturations in cotton. Indian. J. Agric. Sci. 64: 111-113.

Pascual, B. Bautisia, A. San, Lopez-Galarza, S. Alagarda, J. and Moroto, J.V. 2006. Germination behavior after storage of caper seed. Seed sci. and Technol. 34: 151-159.

Satpal, Duhan, B.S., Joshi, U.N., Godara, A.S., Arya, S. and Neelam. 2015. Response of yield, quality and economics of single cut forage sorghum genotypes to different nitrogen and phosphorus levels. Forage Res. 41(3): 170-175.

Sindhawani, S.K. 1991. Use of certified seeds and its contribution towards productivity. Souvenior, Seminar on Seed Industry in Haryana- Present and Future. September 12-13, 1991, HAU, Hisar.

Singh, D. 2009. Seed quality assessment in artificial and natural aged seed of wheat (Triticum aestivum L.). Ph.D. Thesis CCS Haryana, Hisar Haryana, India.

Soltani, E. Galeshi, S. Kamkar, B. and Akramghaderi, F. 2009. The effect of seed aging on the seedling growth as affected by environmental factors in wheat. Res. J. Envi. Sci. 3(2): 184-192.

Tokas, J, Satpal and Kathwal, R., 2019. Chara faslon me vishele tatvon $\mathrm{ka}$ parbandhan. Kheti, 72(7): 22-24.

Verma, S.S. Verma, U. and Tomer, R.P.S. 2003. Studies on seed quality parameter in deteriorating seeds in brassica (Brassica compestris). Seed Sci. \& Technol. 31: 389-396.

USDA (United States Departement of Agriculture). 2011. United States Department of Agriculture Research Service. Nutrient Database for Standard Reference, Nutrient Data Laboratory, USDA publisher, New York. 


\section{How to cite this article:}

Nisha, S. S. Jakhar, Axay Bhuker and Satpal. 2020. Effect of Priming Treatments to Enhance Seed Quality of Naturally Aged Seed of Forage Sorghum. Int.J.Curr.Microbiol.App.Sci. 9(05): 1555-1563. doi: https://doi.org/10.20546/ijcmas.2020.905.176 